\title{
The Other Side of Social Networks: An Ubuntu Approach
}

\author{
Primrose Hlatshwayo \\ Midlands State University, Faculty of Arts, Communication Skills Centre, Gweru, Zimbabwe
}

Email address:

hlatshwayop@msu.ac.zw

\section{To cite this article:}

Primrose Hlatshwayo. The Other Side of Social Networks: An Ubuntu Approach. International Journal of Language and Linguistics. Vol. 4, No. 4, 2016, pp. 153-156. doi: 10.11648/j.ij11.20160404.13

Received: March 24, 2016; Accepted: June 24, 2016; Published: August 2, 2016

\begin{abstract}
The study provides the results of a content based analysis carried out on one hundred and fifty Face book profiles. Using the tenets of Ubuntu, the study sought to find out if the social networking communities are still abiding to the dictates of Ubuntu. The study revealed that a large percentage of the content on FB shows some elements of Ubuntu and an insignificant percentage of the content on FB shows little or no elements of Ubuntu. The elements of Ubuntu which were observed by the researcher are respect, compassion, love, sharing, tolerance and solidarity. The study revealed that, the need to be accepted, age, people's background and relationships affect the way people behave on FB. The study concludes that Face book is being used by African people to remind, instill and perpetrate the values of Ubuntu in its users.
\end{abstract}

Keywords: Ubuntu, Face Book (FB), Love, Sharing and Respect

\section{Introduction}

The social media has been portrayed as a platform for moral decadency for its users. Chiyadzwa and Dube argue that the technology is posing some threats to the morals of the society as some individuals are causing mayhem without shame on social media [1]. Scholars argue that technology has brought threats to the morals of the African society in different ways like promoting indecent dressing, drug abuse and pornography, assault, robbery and secret cult activities which suggest a careless life style which is in contrary to the values and norms of the African people [2-3]. In the same vein, Fortner believes that any new means of communication alters or shakes a culture [4]. This is also echoed in the technological determinism theory which predicts that technology changes a society but it is up to the members to use the technology to sustain their culture or to embrace another new culture [5]. However, this paper argues that despite all the negative things that have been attributed to the social media, there still remain some traces or elements of good behavior within the social network communities. It is the researcher's belief that there are some African face book users who uphold the values and norms of the African culture. The social media platform has played a positive role in as far as reinforcing, encouraging and perpetrating Ubuntu in its users is concerned.
The tenets of the Ubuntu philosophy were exploited to help in identifying the positive aspects on FB. The researcher argues that platforms such as Face book are not created in a vacuum but are constrained by aspects of their geographical central culture. In a way, the social media is only magnifying a community's existing social behavior that is making peoples activities more visible from an individual, family and community level. Ogbeveon argues that the social media can catalyze close, supportive and civically engaged relationships [6]. Some scholars are of the view that the social networks are not changing people's practices but are changing the extent to which their social practices are recorded. In globalised villages, which are characterized by migration from one city to another and one country to another, African people are said to be a people who are accustomed to living close to each other in the village [7]. Given such a case, it is reasonable enough, to conclude that $\mathrm{FB}$ has managed to facilitate that close proximity which the African people value so much. Lomborg observed that FB encourages its users to connect to people they already know i.e. relationships that used to be articulated and maintained independently of one another in different contexts of everyday social life are now brought together on one social platform [8]. The emergence of new media has shrunk the world into a much smaller interactive space, imitating what is called the 'dare' in Shona, where the African brothers and sisters would meet to discuss 
issues. As such, social media has become a rendezvous for the African people who are scattered all over the world in search of greener pastures.

\section{A Description of the Ubuntu Philosophy}

The study made an attempt to trace the elements or traces of Ubuntu on Social media specifically FB. The paper sought to establish if the social network communities are still living in accordance with traditional values and principles or whether they still adhere to the dictates of Ubuntu 'hunhu'. Ubuntu describes a traditional philosophy that is based on the values of humanness, caring, sharing, respect, compassion and associated values [9]. In the Zimbabwean context, Ubuntu is generally referred to as Hunhu' by the Shona people. Nasbaum refers to Ubuntu as the capacity to in African culture to express companion, reciprocity, dignity, harmony and humanity in the interest of building a community with justice and mutual caring [10]. It is clear that Ubuntu is all about perpetrating and reinforcing good behavior and any normal and functional community, even the cyber communities also enjoy the fruits of Ubuntu, such as harmony and justice. Republic of South Africa Government Gazette cited in Muzvidziwa and Muzvidziwa indicate that;

Ubuntu is simply the movement and feeling of compassion for our fellow human beings in our hearts and the spontaneous desire to act in a caring and compassionate way, in which our selfhood is inspired by a sense of collective belonging [11].

Ubuntu means that people are people through other people: Umuntu ngumuntu ngabantu [11]. It is the researcher's belief that some face book users subscribe to this element of Ubuntu since joining the social network implies that you acknowledge that you are what you are because of other people.

Ubuntu is also referred to as a philosophy of life that is concerned with the reinforcement of unity, oneness and solidarity among the African people [13]. Ubuntu is simply a way of living and being that allows our basic goodness to come forth. The core defining values of Ubuntu are care, compassion, unity, tolerance, respect, closeness, generosity, genuineness, empathy, hospitality, conscience, conformity and sharing

[12]. For Tutu, Ubuntu means generosity, hospitability, friendliness care and compassion [13]. The descriptions provided by these scholars clearly show that Ubuntu prevails on FB as the core business of FB is about making friends and connecting with friends. People whom you interact with on $\mathrm{FB}$ are referred to as friends and one

Can conclude, basing on these descriptions that $\mathrm{FB}$ is basically a phenomenon or a branch of Ubuntu despite its western roots.

\section{Elements of Ubuntu on FB}

The researcher analyzed content from one hundred and fifty FB individual profiles The content ranged from pictures, videos, likes, statuses, comics, links and comments posted by the selected individuals. The elements of Ubuntu which were identified are interconnectedness, love, respect, sharing, compassion, hospitality, tolerance and communitarianism.

\subsection{Interconnectedness and Love}

This is shown through the random greetings by friends on the social network platform. This reveals the desire in humans to want to know how the other person is and how they have been. From the research carried out, the study reveals that ninety percent of the posts on FB profiles that were selected reveal some elements of interconnectedness and love. This was basically taken from people's status, messages and comments posted on the platform. Greeting posts are common on face book walls especially in the morning. Individual friends will send messages and this truly show elements of hunhu in FB users or amongst the FB community. Asking the other people if they slept well or if they spent their day well shows empathy and care. FB itself has a programme which reminds individuals about their friends' special events such as birthdays and anniversaries. If it's a certain individual's birthday, they are assured of receiving a dozen birthday messages. This alone promotes love and it is a sigh which shows us that love still abides on social media platforms. The researcher also noted that mostly, those people who are on FB are the ones who never forget a friend's special event but those who are not on FB usually forget. However, ten percent of the posts on the platform show some elements of hate and lack of oneness

\subsection{Sharing and Compassion}

FB has provided a platform for individuals to share what they have. This is a situation where people use or enjoy something jointly with others and compassion is understood as the humane quality of understanding the suffering of others and wanting to do something about it. Gelfand posits that sharing is widespread amongst the African people. Gelfand believes that a good person who has 'hunhu' or values ubuntu should always share what they have with their family and friends. [7]. This element is also one of the most emphasized values of Ubuntu. From the analysis carried out, it is observed that ten percent of the content on FB reveals some elements of sharing and compassion. This is shown through Pictures, messages, statuses videos and comments on the platform. This trend of sharing ideas is clearly noticed on a group called 'Pregnancy and health tips' a group that was created by pregnant women and those who are not in order to assist one another emotionally, financially and with ideas. The aspect of sharing ideas is also supported by the network itself, such that whenever an in individual receives a post, they are always given an option to share, in this case one will simply click on the word share and the message will be received by all your friends. The word share is a common word on FB which is practiced on a daily basis by members of its community. 


\subsection{Respect}

Another element of Ubuntu as noted from the descriptions of Ubuntu earlier on is respect. The researcher observed that the community upholds the Ubuntu values by respecting one another. This is shown by the use of polite language, avoiding posting harsh material and pornographic material. Many groups formed on this platform have rules or codes of conduct which emphasize the use of polite language, upholding the values of respect and avoiding the use of vulgar language. From the content based analyses carried out, it was noted that ninety percent of the material posted on the selected individual profiles revealed the element of respect. The videos, pictures, jokes, messages, likes and comments analyzed, show that people on the FB platform have respect for one another. However, the researcher noted that only ten percent of the posts analyzed showed some lack of respect on some individuals. The researcher noted the use of vulgar words, harsh words, hate speech, pornographic pictures and videos and a few individuals liked indecent material like pictures of nude people etc.

\subsection{Empathy, Oneness and Solidarity}

Empathy, oneness and solidarity show the ubuntu idea that 'I am because you are' and translated in Shona as, 'munhu munhu nevamwe vanhu'. From the content based analysis carried out by the researcher, it was observed that ten percent of all the messages posted revealed empathy, twenty percent of the messages revealed oneness and ten percent of the entire posts were of solidarity nature. Some of the reasons behind these actions are political. The political situation is unstable and there is an economic meltdown. People are not given the platform to air out their views in public platforms as such they do so through the social media. People speak with one voice on FB, especially on issues to do with the Zimbabwean economy; people are showing support to political activists and feeling for those that were victimized. One democratic campaign that has received support on FB is the 'This flag -IflegiLeyi-MurezaUzu'. However, such messages are not well received by everyone and not everyone has the ability to feel for others as a result, some people are judgmental and some texts on FB have shown division and lack of oneness and such messages constitute five percent of the total posts which the researcher analyzed. Lack of empathy, oneness and solidarity in most cases is caused by political affiliations; people on FB belong to different political parties.

\subsection{Tolerance}

This refers to the ability in humans to recognize and respect the beliefs and practices of others. Membership on face book is not based on racial or ethnic background; as such people from different ethnic and racial backgrounds constitute the face book society. However, members have a choice to form some communities based on status, race, education, profession to mention just a few. The platform has rarely been used as a platform to show disrespect or discrimination towards other members. People from different backgrounds have managed to share their views, get assistance, share some links, advertise what they sell and showcase their cultures without anybody prohibiting them or showing disrespect. From the content based analysis done, the study reveals that ninety percent of the texts that were analyzed showed some elements of tolerance. However, the analysis shows that ten percent of all the messages that the researcher stumbled upon revealed some form of discrimination, individualism and lack of solidarity. It was observed that ignorance is the major contributing factor of discriminatory content. In most cases individuals sent discriminatory messages or posts without knowing the impact. In some cases individuals will apologize after noticing that the posts they would have sent were discriminatory in nature.

\section{Explaining People's Behavior on FB}

The researcher observed that there are some reasons which make people to respect one another. The need to be accepted forces people to respect one another, those people who display indecent behaviour, use vulgar language and liking indecent material are usually blocked. Again, the researcher noted that age is also another factor that impacts on how people behave on social media platforms. The sample of profile pages selected was of individuals ranging from the age of twenty five up to fifty. These are adults and some of the individuals have children who are also on FB. As such, they are forced in a way to behave themselves because they do not want their children to fall in the same trap. The researcher assumes that younger people especially teenagers are the ones who are more into pornography than the older generation because they are experimenting.

Moreover, the researcher also noted that most of the individuals selected have very close relatives as their friends on FB, for example spouses, siblings, in-laws, aunts and in most cases real friends. It is obvious that individuals would not want their close relations to see their negative sides; hence they uphold the elements of ubuntu.

The researcher also noted that there are also some factors which make individuals behave indecently on social media. Ignorance makes individuals not to abide by the dictates of ubuntu, for example some people will just post some material not knowing that once they do that, the material will be seen by anyone who accesses their profiles. Again, in some cases some individuals will just click 'like' on certain materials or links without fully understanding the materials and the implications of clicking' like' on FB.

Moreover, the need to belong to a certain group of people makes individuals behave indecently, for example associating with a group of people who like indecent material like pornography and hate speech, as such one will just tarnish his or her name by association. That is why there are some individuals who stipulate the kind of people they want to be friends with on FB, more like ground rules. 


\section{Conclusion}

The research concludes that social media platforms like Face book can actually be utilized as tools to uphold the African culture as many researchers suggest that it is coming to its extinction because of the Western technology. The study revealed that many African people have displayed some traits of Ubuntu, like respect, compassion, sharing, tolerance and interconnectedness. Face book discourse by African members has revealed that the platform is there to uphold, reinforce and to remind people of some of these Ubuntu elements, however, the researcher observed that, many people especially the older generation are failing to accept FB as a legitimate platform for connecting with friends and sharing ideas. A large chunk of posts analyzed by the researcher shows some elements of Ubuntu. However a smaller portion of the material analyzed shows lack of Ubuntu, for example some of the posts show discrimination, hatred, division lack of respect and selfishness. The study concludes that a need to be accepted, relationships, politics, age, ignorance and lack of maturity are the contributing factors to the people's behavior on FB. It is the researcher's belief, that the moment the older African generation accepts FB as a legitimate and harmless social networking platform; the Ubuntu traits will become more prevalent on FB.

\section{References}

[1] I. F. Chiyadzwa, and E, Dube, 'Bedroom Politics, the social media ulcer: An application of the hunhu, ubuntu Philosophy in light of the technological determinism and cultural imperialism theory', Journal of Humanities and Social Sciences, 19121-127, 2014.
[2] O. Nkechi and G. C. Nche, 'The moral implication of social media phenomenon in Nigeria', Mediterranean Journal of Social Sciences, 15, 2231-2237, 2014.

[3] A. N. Lumun, 'The Effects of Western Technology on African Cultural Values', International Journal Of Humanities and Social Sciences, 5 41-46, 2015.

[4] R. S. Fortner, Communication, Media and Identity, Rowan and Littlefield, London, 2007.

[5] M. Solo-Anaeto. And B. Jacobs, 'Exploring social media as channels for sustaining African Culture', International Journal of Social Sciences and Humanities, 4 (1) 37-48, 2013.

[6] L. Ogbovern, '3Qs: The social impact of social networks', News@Northeastern University, 3 October, 2012.

[7] M. Gelfand, The Genuine Shona: A Survival Value of an African Culture: Mambo Press: Gweru, 1973.

[8] S. Lomborg, Social Media, Social Genres: Making Sense of the Ordinary,: Routledge: New York, 2014.

[9] J. Broodryk, "Ubuntu: African Life Coping Skills: Theory and Practice."

[10] B. Nussbaum, "African Culture and Ubuntu Reflections of a South African in America," World Business Academy 17, 1, 2003.

[11] N. Muzvidziwa, And I. Muzvidziwa, 'Ubuntu and Discipline in African Schools' Journal of Dharma 37 (1) 27-42, 2003.

[12] F. M. Mulaudzi, M. Libster, ands. Phiri, "Suggestions for creating a welcoming Nursing Community: Ubuntu, Cultural Diplomacy and Mentoring," International Journal of Human Caring, 13, 2 (2009), 47-59. 